\title{
Testing $S$-duality with non-orientable surfaces
}

\author{
Siye $\mathbf{W u}^{* \dagger}$ \\ Department of Mathematics, National Tsing Hua University, Hsinchu 30013, Taiwan \\ E-mail: swu@math.nthu.edu.tw
}

Kapustin and Witten showed that a twisted version of $N=4$ gauge theory in four dimensions compactifies to a two-dimensional sigma-model whose target space is the Hitchin moduli space. In this talk, I consider the reduction of the gauge theory on a four dimensional orientable spacetime manifold which is not a global product of two surfaces but contains embedded non-orientable surfaces. The low energy theory is a sigma-model on a two dimensional worldsheet whose boundary components end on branes constructed from the Hitchin moduli space associated to a nonorientable surface. I will also compare the discrete topological fluxes in four and two dimensional theories and verify the mirror symmetry on branes as predicted by the $S$-duality in gauge theory. This provides another non-trivial test of $S$-duality using reduction along possibly non-orientable surfaces. Finally, I consider the quantisation of the Hitchin moduli space from a non-orientable surface as an example of quantisation via branes and mirror symmetry.

The 39th International Conference on High Energy Physics (ICHEP2018)

4-11 July, 2018

Seoul, Korea

\footnotetext{
*Speaker.

${ }^{\dagger}$ Supported in part by grant No. 106-2115-M-007-005-MY2 from MOST and the NCTS (physics) of Taiwan.
} 


\section{Topological sectors of 4-dimensional gauge theory}

We consider 4-dimensional gauge theory with a gauge group $G$ which is a connected compact semisimple Lie group. Electric charges of a fundamental particle (or field) are irreducible representations of $G$, classified by the weight vectors in the positive Weyl chambre. On the other hand, magnetic monopoles, in classical gauge theory, are from gauge potentials (or connections) that are singular in space. Given a homomorphism from $\mathrm{U}(1)$ to $G$, there is a Yang-Mills connection induced by the Dirac monopole in $\mathrm{U}(1)$ gauge theory of charge 1 . Therefore the magnetic charges are elements in $\operatorname{Hom}(\mathrm{U}(1), G)$ up to conjugations in $G$ (to account for gauge equivalence). The latter corresponds to the coweight vectors in the positive chambre. At the quantum level, magnetic charges describe collective excitations whereas electric charges are of elementary excitations.

In addition to these charges, a 4-dimensional gauge theory has discrete fluxes which belong to finite Abelian groups. After Wick rotation, the spacetime has Euclidean signature and can be chosen as a compact orientable 4-manifold $X$. Classical gauge theory builds upon the geometry of principal $G$-bundles over $X$. These bundles are classified topologically by characteristic classes in $H^{4}\left(X, \pi_{3}(G)\right)$ and $H^{2}\left(X, \pi_{1}(G)\right)$. When $G$ is simple, such as $\mathrm{SU}(n)$ with $n \geq 2$, we have $\pi_{3}(G) \cong \mathbb{Z}$, and $H^{4}\left(X, \pi_{3}(G)\right) \cong \mathbb{Z}$ contains the instanton numbers. Since $\pi_{1}(G)$ is finite Abelian, the group $H^{2}\left(X, \pi_{1}(G)\right)$ is necessarily torsion and it contains the discrete fluxes of 't Hooft [1].

When $X$ has a splitting of space and time, i.e., when $X=T^{1} \times Y$, where $T^{1}$ is a circle in the time direction and $Y$ is a compact orientable spatial 3-manifold, there is a decomposition

$$
H^{2}\left(X, \pi_{1}(G)\right) \cong H^{2}\left(Y, \pi_{1}(G)\right) \oplus H^{1}\left(Y, \pi_{1}(G)\right) .
$$

An element $m \in H^{2}\left(Y, \pi_{1}(G)\right)$ classifies the topology of the $G$-bundle over a time slice $Y$ and is called the discrete magnetic flux. A fixed $m$ determines a well defined sector both classically and quantum mechanically. On the contrary, an element $a \in H^{1}\left(Y, \pi_{1}(G)\right) \cong H^{1}\left(Y, H^{1}\left(T^{1}, \pi_{1}(G)\right)\right)$ contains the information of the entire time interval and can not be fixed consistently.

Instead, we interpret discrete electric fluxes as the momenta of discrete translations on field configurations when the centre $Z(G)$ of $G$ is non-trivial. Recall that $Z(G)$ is also a finite Abelian group. We claim that $H^{1}(Y, Z(G))$ is a discrete symmetry in the gauge theory. An element $g \in H^{1}(Y, Z(G))=\operatorname{Hom}\left(\pi_{1}(Y), Z(G)\right)$ modifies the holonomy of a connection $A$ along a loop $\gamma$ in $Y$ by $g([\gamma]) \in Z(G)$, where $[\gamma] \in \pi_{1}(Y)$ is the class represented by $\gamma$ in the fundamental group. This procedure preserves the curvature (or field strength) and hence the classical action. The quantum Hilbert space is then a representation of $H^{1}(Y, Z(G))$ and decomposes according to the types of its irreducible representations. So the quantum theory consists of sectors labelled by $e \in H^{1}(Y, Z(G))^{\vee}$, where for any Abelian group $A$, the Pontryagin dual $A^{\vee}:=\operatorname{Hom}(A, \mathrm{U}(1))$ is the group of characters of $A$. The elements $e$ are the discrete electric fluxes.

When $G$ is exchanged with its Langlands dual ${ }^{L} G$ or its magnetic group, so do the weight and coweight lattices and the sets of electric and magnetic charges [2]. In adition, we have

$$
H^{1}(Y, Z(G))^{\vee} \cong H^{2}\left(Y, \pi_{1}(G)\right), \quad H^{2}\left(Y, \pi_{1}(G)\right) \cong H^{1}\left(Y, Z\left({ }^{L} G\right)\right)^{\vee}
$$

from Poincaré duality. So the discrete electric fluxes in the $G$-theory are the discrete magnetic fluxes in the ${ }^{L} G$-theory, and vice versa. This is consistent with the electric-magnetic duality (or $S$-duality) proposed by [3]. 


\section{Reduction to 2-dimensions along orientable surfaces}

In [4], Kapustin and Witten considered the reduction of a twisted $N=4$ supersymmetric gauge theory on the 4-manifold $X=\Sigma \times C$ to a sigma-model whose worldsheet is $\Sigma$ along a compact orientable surface $C$ of genus $g(C)>1$. The target space of the low energy theory is the Hitchin moduli space $\mathcal{M}_{\mathrm{H}}(C, G)$, which is a hyper-Kähler manifold with complex structures $I, J, K$ and Kähler forms $\omega_{I}, \omega_{J}, \omega_{K}$ following the notations of [4].

When the worldsheet has a splitting $\Sigma=T^{1} \times S^{1}$, so does the 4-dimensional spacetime $X=$ $T^{1} \times Y$, in which $Y=S^{1} \times C$. In gauge theory, we write the discrete magnetic flux $m=m_{0}+m_{1}$ and the discrete electric flux $e=e_{1}+e_{0}$ according to the decompositions

$H^{2}\left(Y, \pi_{1}(G)\right) \cong H^{2}\left(C, \pi_{1}(G)\right) \oplus H^{1}\left(C, \pi_{1}(G)\right), \quad H^{1}(Y, Z(G))^{\vee} \cong H^{1}(C, Z(G))^{\vee} \oplus H^{0}(C, Z(G))^{\vee}$.

In the sigma-model, $m_{0} \in \pi_{0}\left(\mathcal{M}_{\mathrm{H}}(C, G)\right)$ labels the connected component in which the string propagates while $m_{1} \in \pi_{1}\left(\mathcal{M}_{\mathrm{H}}(C, G)\right)$ is the winding of the string. On the other hand, $e_{1}$ is a discrete momentum of the symmetry group $H^{1}(C, Z(G))$ acting on $\mathcal{M}_{\mathrm{H}}(C, G)$, whereas $e_{0}$ labels the flat $B$-field on $\mathcal{M}_{\mathrm{H}}(C, G)$ coupled to the sigma-model [4].

Electric-magnetic duality in four dimensions, which is believed to be exact in $N=4$ gauge theories, reduced to mirror symmetry in two dimensions [5, 6, 4]. Indeed, $\mathcal{M}_{\mathrm{H}}(C, G)$ and $\mathcal{M}_{\mathrm{H}}\left(C,{ }^{L} G\right)$ are mirrors to each other [7, 8] in the sense of [9]. With the exchange of $G$ and ${ }^{L} G$, the roles of $m_{0}$ and $e_{0}, m_{1}$ and $e_{1}$, also interchange. The mirror correspondence of branes on $\mathcal{M}_{\mathrm{H}}(C, G)$ and $\mathcal{M}_{\mathrm{H}}\left(C,{ }^{L} G\right)$ explains much of the geometric Langlands programme [4].

\section{Reduction along possibly non-orientable surfaces}

In [10], we consider the gauge theory on a 4-manifold which is not a global product but contains embedded non-orientable surface $C^{\prime}$. Let $\pi: C \rightarrow C^{\prime}$ be the orientation double cover: there is a free $\mathbb{Z}_{2}$ action on $C$ and the quotient is $C^{\prime}$. For example, $C=S^{2}$ and $C^{\prime}=C / \mathbb{Z}_{2}=\mathbb{R} P^{2}$. But we will assume $C^{\prime}$ is a connected sum of $g\left(C^{\prime}\right)>2$ copies of $\mathbb{R} P^{2}$. For the worldsheet, we take an orientable surface $\tilde{\Sigma}$ with an orientation-reversing $\mathbb{Z}_{2}$-action. The quotient $\Sigma=\tilde{\Sigma} / \mathbb{Z}_{2}$ is a surface whose boundary $\partial \Sigma$ is the fixed-point set of $\mathbb{Z}_{2}$ on $\tilde{\Sigma}$, which is assumed to be non-empty. For example, $\Sigma$ is a disc for $\tilde{\Sigma}=S^{2}$ with a reflection along the equator. The 4-manifold $X=\tilde{\Sigma} \times{ }_{\mathbb{Z}_{2}} C$ (quotient by the diagonal action) is a smooth orientable 4-manifold without boundary [10]. There is a projection $\pi_{X}: X \rightarrow \Sigma$ by forgetting $C$. The inverse image $\pi_{X}^{-1}(\sigma)$ is a copy of $C$ if $\sigma$ is in the interior of $\Sigma$ but is $C^{\prime}$ if $\sigma \in \partial \Sigma$. So $X$ contains a $\partial \Sigma$-family of non-orientable surfaces $C^{\prime}$.

Hitchin's equations make sense on a non-orientable surface $C^{\prime}$ and the moduli space $\mathcal{M}_{\mathrm{H}}\left(C^{\prime}, G\right)$ of solutions modulo gauge equivalence is introduced and studied in details in [11]. There is a map $p: \mathcal{M}_{\mathrm{H}}\left(C^{\prime}, G\right) \rightarrow \mathcal{M}_{\mathrm{H}}(C, G)$ by pulling back fields from $C^{\prime}$ to $C$. The image $\mathcal{N}(C, G)$ of $p$ is contained in the $\mathbb{Z}_{2}$-invariant part $\mathcal{M}_{\mathrm{H}}(C, G)^{\mathbb{Z}_{2}}$ of $\mathcal{M}_{\mathrm{H}}(C, G)$; the latter is Lagrangian in $\omega_{I}, \omega_{K}$ and holomorphic in $J$. On the smooth part, $p: \mathcal{M}_{\mathrm{H}}\left(C^{\prime}, G\right) \rightarrow \mathcal{N}(C, G)$ is a finite regular $Z(G)_{[2]}$-cover. Here $A_{[2]}=\{a \in A: 2 a=0\}$ is the 2-torsion subgroup for any Abelian group $A$.

In the limit of large $\tilde{\Sigma}$ (or $\Sigma$ ) and small $C$ (or $C^{\prime}$ ), the same $N=4$ gauge theory on $X$ reduces to a sigma-model of target space $\mathcal{M}_{\mathrm{H}}(C, G)$ on $\Sigma$ with the boundary $\partial \Sigma$ living on branes [10]. Among the bosonic variables are the maps $u: \Sigma \rightarrow \mathcal{M}_{\mathrm{H}}(C, G)$ and $u^{\prime}: \partial \Sigma \rightarrow \mathcal{M}_{\mathrm{H}}\left(C^{\prime}, G\right)$ satisfying 
$p \circ u^{\prime}=\left.u\right|_{\partial \Sigma}$. For each $e_{2} \in\left(Z(G)_{[2]}\right)^{\vee}$, we get a flat Chan-Paton bundle $\ell^{e_{2}}=\mathcal{M}_{\mathrm{H}}\left(C^{\prime}, G\right) \times_{e_{2}} \mathbb{C}$ over $\mathcal{N}(C, G)$. On the other hand, there is a decomposition $\mathcal{M}_{\mathrm{H}}\left(C^{\prime}, G\right)=\bigsqcup_{m_{2} \in \pi_{1}(G) / 2 \pi_{1}(G)} \mathcal{M}_{\mathrm{H}}^{m_{2}}\left(C^{\prime}, G\right)$ according to the topological types of $G$-bundles over $C^{\prime}$, and we let $\mathcal{N}^{m_{2}}(C, G)=p\left(\mathcal{M}_{\mathrm{H}}^{m_{2}}\left(C^{\prime}, G\right)\right)$. Then $\mathcal{B}^{e_{2}, m_{2}}=\left(\mathcal{N}^{m_{2}}(C, G), \ell^{e_{2}}\right)$ is a brane on $\mathcal{M}_{\mathrm{H}}(C, G)$ of type $(A, B, A)$. The low energy theory on $\Sigma$ contains sectors whose boundary conditions are defined by $\mathcal{B}^{e_{2}, m_{2}}$.

Consider the case $\tilde{\Sigma}=T^{1} \times S^{1}$ with $\mathbb{Z}_{2}$ acting on $S^{1}$ by reflection. Then $\Sigma$ is a cylinder with two time-like boundary circles. The sigma-model is about the propagation of an open string whose boundary points are constrained on branes. By homotopy calculations [10], the relative winding of the open string is $m_{1} \in H^{1}\left(C, \pi_{1}(G)\right) / \pi^{*} H^{1}\left(C^{\prime}, \pi_{1}(G)\right)$. The presence of branes reduces the $H^{1}(C, Z(G))$ symmetry to $\pi^{*} H^{1}\left(C^{\prime}, Z(G)\right)$ and the discrete momenta are $e_{1} \in \pi^{*} H^{1}\left(C^{\prime}, Z(G)\right)^{\vee}$. So the sectors of the 2-dimensional theory are labelled by $m_{1}, e_{1}, m_{2}, e_{2}$. The absence of $m_{0}$ is because only the component $\mathcal{M}_{\mathrm{H}}^{m_{0}=0}(C, G)$ supports the branes whereas the absence of $e_{0}$ is due to an anomaly-free condition [10] much like the Freed-Witten condition [12] for untwisted strings.

The 4-dimensional spacetime is $X=T^{1} \times Y$, where $Y=S^{1} \times_{\mathbb{Z}_{2}} C$ is a smooth compact orientable 3-manifold without boundary. The sets of discrete electric and magnetic fluxes in the gauge theory are given by the exact sequences [10]

$$
\begin{gathered}
0 \rightarrow \frac{H^{1}\left(C, \pi_{1}(G)\right)}{\pi^{*} H^{1}\left(C^{\prime}, \pi_{1}(G)\right)} \rightarrow H^{1}\left(Y, \pi_{1}(G)\right) \rightarrow\left(\pi_{1}(G) / 2 \pi_{1}(G)\right)^{\oplus 2} \rightarrow 0, \\
0 \rightarrow\left(\pi^{*} H^{1}\left(C^{\prime}, Z(G)\right)\right)^{\vee} \rightarrow H^{1}(Y, Z(G))^{\vee} \rightarrow\left(Z(G)_{[2]}^{\oplus 2}\right)^{\vee} \rightarrow 0 .
\end{gathered}
$$

This matches the low energy data: we see the absence of $m_{0}, e_{0}$, the relative windings $m_{1}$, the discrete momenta $e_{1}$, and two copies of $m_{2}, e_{2}$ because an open string has two end points.

As usual, $S$-duality exchanging $G$ and ${ }^{L} G$ reduces to mirror symmetry, interchanging the roles of $m_{1}$ and $e_{1}, m_{2}$ and $e_{2}$. The latter is made possible by the isomorphisms

$$
\frac{H^{1}\left(C, \pi_{1}(G)\right)}{\pi^{*} H^{1}\left(C^{\prime}, \pi_{1}(G)\right)} \cong\left(\pi^{*} H^{1}\left(C^{\prime}, Z\left({ }^{L} G\right)\right)\right)^{\vee}, \quad \pi_{1}(G) / 2 \pi_{1}(G) \cong\left(Z\left({ }^{L} G\right)_{[2]}\right)^{\vee},
$$

etc. The brane $\mathcal{B}^{e_{2}, m_{2}}$ in the original theory and ${ }^{L_{\mathcal{B}} m_{2}, e_{2}}$ in the dual theory are related by a fibrewise Fourier-Mukai transform [10] between branes on $\mathcal{M}_{\mathrm{H}}(C, G)$ and $\mathcal{M}_{\mathrm{H}}\left(C,{ }^{L} G\right)$ that are dual special Lagrangian fibrations. This provides another non-trivial test of $S$-duality with dimensional reduction along non-orientable surfaces, using the properties of the moduli space $\mathcal{M}_{\mathrm{H}}\left(C^{\prime}, G\right)$.

\section{Adjustments for full generality}

The above consideration, while sufficient in many circumstances, is not yet completely accurate in the most general setting. It can happen that the electric and magnetic fluxes can not be simultaneously fixed because the discrete symmetry $H^{1}(Y, Z(G))$ may change the topology of the $G$-bundle over $Y[13,10]$. More precisely, an element $g \in H^{1}(Y, Z(G))$ changes the discrete magnetic flux $m$ to $m+\delta(g)$, where $\delta_{Y}$ is the connecting homomorphism in the long exact sequence

$$
\cdots \rightarrow H^{1}(Y, Z(\widetilde{G})) \rightarrow H^{1}(Y, Z(G)) \stackrel{\delta_{Y}}{\longrightarrow} H^{2}\left(Y, \pi_{1}(G)\right) \rightarrow H^{2}(Y, Z(\widetilde{G})) \rightarrow \cdots,
$$

where $\widetilde{G}$ is the universal cover group of $G$. This detail has been overlooked in the past literature because the map $\delta_{Y}$ is zero in many cases, such as when $H_{1}(Y)$ is torsion-free (e.g., if $Y=S^{1} \times C$ 
as in $\S 2$ ) or when the short exact sequence $0 \rightarrow \pi_{1}(G) \rightarrow Z(\widetilde{G}) \rightarrow Z(G) \rightarrow 0$ of $Z(\widetilde{G})$ splits. But for the 3-manifold $Y=S^{1} \times{ }_{\mathbb{Z}_{2}} C$ in $\S 3$, the map $\delta_{Y}$ can be non-zero.

A classical symmetry, if not anomalous at the quantum level, becomes part of the automorphism group of the quantum operator algebra. If the symmetry group does not act on the quantum Hilbert space, which is an irreducible representation of the operator algebra, the symmetry is said to be broken to the subgroup which does act on the Hilbert space. Inner automorphisms are always in the unbroken subgroup, but typically, an outer automorphism sends an irreducible representation to a different one. In our case, since the Hilbert space is labelled by $m$, the unbroken subgroup in $H^{1}(Y, Z(G))$ is $\operatorname{ker}\left(\delta_{Y}\right)$, and consequently, the discrete electric fluxes are in its character group $\mathfrak{e}(Y, G):=\operatorname{ker}\left(\delta_{Y}\right)^{\vee}$. On the other hand, the discrete magnetic fluxes should label non-isomorphic quantum theories, and they are in $\mathfrak{m}(Y, G):=H^{2}\left(Y, \pi_{1}(G)\right) / \operatorname{im}\left(\delta_{Y}\right)=\operatorname{coker}\left(\delta_{Y}\right)$. For $Y=S^{1} \times_{\mathbb{Z}_{2}} C$, the sets $\mathfrak{e}(Y, G), \mathfrak{m}(Y, G)$ of these rectified fluxes can be computed explicitly and they are different from $H^{1}(Y, Z(G))^{\vee}, H^{2}\left(Y, \pi_{1}(G)\right)$. Under $S$-duality, there are isomorphisms $\mathfrak{e}(Y, G) \cong \mathfrak{m}\left(Y,{ }^{L} G\right)$, $\mathfrak{m}(Y, G) \cong \mathfrak{e}\left(Y,{ }^{L} G\right)$, exchanging the rectified electric and magnetic fluxes [10].

In the low energy theory, although each $\mathcal{M}_{\mathrm{H}}^{m_{2}}\left(C^{\prime}, G\right), m_{2} \in \pi_{1}(G) / 2 \pi_{1}(G)$, is expected to be connected, it is not preserved by the full covering group $Z(G)_{[2]}[13,10]$. In fact, $g \in Z(G)_{[2]}$ sends $m_{2}$ to $m_{2}+\delta_{\mathbb{Z}_{2}}(g)$, where $\delta_{\mathbb{Z}_{2}}: Z(G)_{[2]} \rightarrow \pi_{1}(G) / 2 \pi_{1}(G)$ is the connecting homomorphism of a similar long exact sequence (replacing $Y$ by $B \mathbb{Z}_{2}=\mathbb{R} P^{\infty}$ ). The map $\delta_{\mathbb{Z}_{2}}$ is zero when all elements in $Z(\widetilde{G})$ are of odd order or when the above short exact sequence of $Z(\widetilde{G})$ splits. So the branes are $\mathcal{B}^{\bar{e}_{2}, \bar{m}_{2}}$, where $\bar{e}_{2}$ in $\mathfrak{e}\left(\mathbb{Z}_{2}, G\right):=\operatorname{ker}\left(\delta_{\mathbb{Z}_{2}}\right)^{\vee}$ defines a flat Chan-Paton line bundle over the worldvolume $\mathcal{N}^{\bar{m}_{2}}(C, G)$ that depends only on the cosets $\bar{m}_{2}$ in $\mathfrak{m}\left(\mathbb{Z}_{2}, G\right):=\operatorname{coker}\left(\delta_{\mathbb{Z}_{2}}\right)$. With the above modifications, the 4- and 2-dimensional data still match just as in $\S 3$. Under $S$-duality, there are isomorphisms $\mathfrak{e}\left(\mathbb{Z}_{2}, G\right) \cong \mathfrak{m}\left(\mathbb{Z}_{2},{ }^{L} G\right), \mathfrak{m}\left(\mathbb{Z}_{2}, G\right) \cong \mathfrak{e}\left(\mathbb{Z}_{2},{ }^{L} G\right)$. So the twisting by Chan-Paton bundles on one side is mirror to displacements of worldvolumes on the dual side. With the rectified discrete fluxes, the mirror of $\mathcal{B}^{\bar{e}_{2}, \bar{m}_{2}}$ is ${ }_{\mathcal{B}} \bar{B}^{\bar{m}_{2}, \bar{e}_{2}}$. We refer the reader to [10] for details.

\section{An example of quantisation via branes and mirror symmetry}

To quantise a symplectic manifold $(M, \omega)$ via branes [14], one needs a complexification $M^{\mathbb{C}}$ with an anti-holomorphic involution fixing $M$. There is also a holomorphic symplectic form $\omega^{\mathbb{C}}$ on $M^{\mathbb{C}}$ such that $\operatorname{Re}\left(\omega^{\mathbb{C}}\right)=\omega$ on $M$. The $\mathbb{Z}_{2}$-action on $M^{\mathbb{C}}$ lifts to a line bundle $\ell$ and preserves its connection with curvature $\operatorname{Re}\left(\omega^{\mathbb{C}}\right) / \sqrt{-1}$. We then have a space-filling coisotropic brane $\mathcal{B}_{\mathrm{cc}}$ in the $A$-model on $M^{\mathbb{C}}$ with symplectic form $\operatorname{Im}\left(\omega^{\mathbb{C}}\right)$. A trivial or flat line bundle on $M$ defines a Lagrangian $A$-brane $\mathcal{B}_{0}$. The quantisation of $(M, \omega)$ is then $\operatorname{Hom}\left(\mathcal{B}_{\mathrm{cc}}, \mathcal{B}_{0}\right)$ [14]. In the $B$-model on the mirror of $M^{\mathbb{C}}$, the quantum Hilbert space is $\operatorname{Ext}\left(\mathcal{B}_{\mathrm{cc}}^{\vee}, \mathcal{B}_{0}^{\vee}\right)$, where $\mathcal{B}^{\vee}$ is the dual of $\mathcal{B}$ [15].

To quantise the Hitchin moduli space $\mathcal{M}_{\mathrm{H}}\left(C^{\prime}, G\right)$ with a non-orientable surface $C^{\prime}$, we need to generalise the above setting [10]: $\mathcal{M}_{\mathrm{H}}\left(C^{\prime}, G\right)$ maps to $\mathcal{M}_{\mathrm{H}}(C, G)^{\mathbb{Z}_{2}}$ in the complexification $\mathcal{M}_{\mathrm{H}}(C, G)$ by a local diffeomorphism which is Kähler with respect to $\omega_{J}$ [11]. A line bundle $\ell$ over $\mathcal{M}_{\mathrm{H}}(C, G)$ whose curvature is $\omega_{J} / \sqrt{-1}$ defines $\mathcal{B}_{\mathrm{cc}}$ [13]. The quantisation of $\mathcal{M}_{\mathrm{H}}^{m_{2}}\left(C^{\prime}, G\right)$ is a sum of $\mathcal{H}^{\bar{e}_{2}, \bar{m}_{2}}=\operatorname{Hom}\left(\mathcal{B}_{\mathrm{cc}}, \mathcal{B}^{\bar{e}_{2}, \bar{m}_{2}}\right)$ over $\bar{e}_{2} \in \mathfrak{e}\left(\mathbb{Z}_{2}, G\right)$ in the $A$-model on $\mathcal{M}_{\mathrm{H}}(C, G)$ with $\omega_{K}$. By mirror symmetry, $\mathcal{H}^{\bar{e}_{2}, \bar{m}_{2}}=\operatorname{Ext}\left(\mathcal{B}_{\mathrm{cc}}^{\vee},{ }_{\mathcal{B}} \bar{m}_{2}, \bar{e}_{2}\right)$ in the $B$-model on $\mathcal{M}_{\mathrm{H}}\left(C,{ }^{L} G\right)$ with $J[13,10]$.

Like the Hitchin moduli space $\mathcal{M}_{\mathrm{H}}(C, G)$, the moduli space $\mathcal{M}_{\mathrm{H}}\left(C^{\prime}, G\right)$ for non-orientable $C^{\prime}$ should also contain $T^{*} \mathcal{M}_{\text {flat }}\left(C^{\prime}, G\right)$ as a dense open set [10]. Here $\mathcal{M}_{\text {flat }}\left(C^{\prime}, G\right)$ is the moduli space 
of flat $G$-connections on $C^{\prime}$. The component $\mathcal{M}_{\text {flat }}^{m_{2}}\left(C^{\prime}, G\right)$ with topological type $m_{2}$ is connected [17] and has an action of $\operatorname{ker}\left(\delta_{\mathbb{Z}_{2}}\right)$ [13]. Using the real polarisation of $T^{*} \mathcal{M}_{\text {flat }}\left(C^{\prime}, G\right), \mathcal{H}^{\bar{e}_{2}, \bar{m}_{2}}$ is the space of wave functions on $\mathcal{M}_{\text {flat }}^{m_{2}}\left(C^{\prime}, G\right)$ that transform according to $\bar{e}_{2}$ under $\operatorname{ker}\left(\delta_{\mathbb{Z}_{2}}\right)$.

\section{References}

[1] G. 't Hooft, A property of electric and magnetic flux in non-abelian gauge theories, Nucl. Phys. B153 (1979) 141-160

[2] P. Goddard, J. Nuyts and D.I. Olive, Gauge theories and magnetic charge, Nucl. Phys. B125 (1977) $1-28$

[3] C. Montonen and D.I. Olive, Magnetic monopoles as gauge particles?, Phys. Lett. 72B (1977) $117-120$

[4] A. Kapustin and E. Witten, Electric-magnetic duality and the geometric Langlands program, Commun. Number Theory Phys. 1 (2007) 1-236, arXiv: hep-th/ 0604151

[5] M. Bershadsky, A. Johansen, V. Sadov and C. Vafa, Topological reduction of 4D SYM to 2D $\sigma$-models, Nucl. Phys. B448 (1995) 166-186, arXiv: hep-th/9501096

[6] J.A. Harvey, G. Moore and A. Strominger, Reducing S-duality to T-duality, Phys. Rev. D52 (1995) 7161-7167, arXiv:hep-th/9501022

[7] T. Hausel and M. Thaddeus, Mirror symmetry, Langlands duality, and the Hitchin system, Invent. Math. 153 (2003) 197-229, arXiv:math/ 0205236 [math.AG]

[8] R. Donagi and T. Pantev, Langlands duality for Hitchin systems, Invent. Math. 189 (2012) 653-735, arXiv:math/0604617 [math.AG]

[9] A. Strominger, S.-T. Yau and E. Zaslow, Mirror symmetry is T-duality, Nucl. Phys. B479 (1996) 243-259, arXiv: hep-th/9606040

[10] S. Wu, Non-orientable surfaces and electric-magnetic duality, J. High Energy Phys. 10 (2018) 169, arXiv:1804:11343 [hep-th]

[11] N.-K. Ho, G. Wilkin and S. Wu, Hitchin's equations on a nonorientable manifold, Commun. Anal. Geom. 26 (2018) 857-886, arXiv: 1211.0746 [math.DG]

[12] D.S. Freed and E. Witten, Anomalies in string theory with $D$-branes, Asian J. Math. 3 (1999) 819-851, arXiv:hep-th/9907189

[13] S. Wu, Twisted character varieties, covering spaces and gerbes, Theor. Math. Phys. 185 (2015) 1769-1788

[14] S. Gukov and E. Witten, Branes and quantization, Adv. Theor. Math. Phys. 13 (2009) 1445-1518, arXiv:0809.0305 [hep-th]

[15] S. Gukov, Quantization via mirror symmetry, Japan. J. Math. 6 (2011) 65-119, arXiv:1011.2218 [hep-th]

[16] S. Wu, Quantization of Hitchin's moduli space of a non-orientable surface, in: Geometric methods in physics, Trends Math., eds. P. Kielanowski et al., pp. 343-363, Birkhäuser/Springer, Cham (2016)

[17] N.-K. Ho and C.-C.M. Liu, Connected components of the space of surface group representations, Inter. Math. Res. Notices 44 (2003) 2359-2371, arXiv:math / 0303255 [math.SG] 